\title{
Uno scrittore francese alla spedizione dei Mille
}

\section{Cecilia Rizza}

\section{(2) OpenEdition}

\section{Journals}

\section{Edizione digitale}

URL: https://journals.openedition.org/studifrancesi/2957

DOI: 10.4000/studifrancesi.2957

ISSN: 2421-5856

\section{Editore}

Rosenberg \& Sellier

\section{Edizione cartacea}

Data di pubblicazione: 1 juillet 2013

Paginazione: $362-368$

ISSN: 0039-2944

\section{Notizia bibliografica digitale}

Cecilia Rizza, «Uno scrittore francese alla spedizione dei Mille», Studi Francesi [Online], 170 (LVII | II)

2013, online dal 30 novembre 2015, consultato il 31 janvier 2023. URL: http://

journals.openedition.org/studifrancesi/2957 ; DOI: https://doi.org/10.4000/studifrancesi.2957

\section{(c) $(1)(9)$}

Creative Commons - Attribuzione - Non commerciale - Non opere derivate 4.0 Internazionale - CC BYNC-ND 4.0

https://creativecommons.org/licenses/by-nc-nd/4.0/ 


\title{
Uno scrittore francese \\ alla spedizione dei Mille
}

\begin{abstract}
Maxime Du Camp's Expédition des Deux Siciles, published in 1861, gives an account of his experiences as a volunteer in the Expedition of the Thousand (Spedizione dei Mille, 1860). Within the wide corpus of writings related to this military campaign, Du Camp's hitherto largely neglected memoir is characterized by its many descriptions of the places, customs and local people (with their prejudices, their lack of education, and their genuine patriotic fervour) which the author encountered while marching north from Pizzo Calabro to Naples. As for the charismatic leader of the expedition, Du Camp does not subscribe to the Garibaldi myth: he acknowledges his achievements and merits, but he also highlights his very substantial limitations as a politician.
\end{abstract}

En 1861 Maxime Du Camp publie L'expédition des Deux Siciles, un ouvrage qui naît de sa participation à l'expédition de Garibaldi et des Mille en Sicile et à Naples. Le livre de Du Camp, peu étudié jusqu'à présent, se caractérise, dans la vaste production italienne et étrangère relative à ce sujet, par l'intérêt que l'auteur donne à la description des lieux et des villages qu'il traverse, et aux mœurs des populations (préjugés, ignorance, enthousiasme patriotique) qu'il rencontre en remontant la péninsule de Pizzo Calabro à Naples. Quant à Garibaldi, Du Camp refuse toute mythisation du personnage dont il reconnait, à côté de grands mérites, des limites assez importantes du point de vue politique.

Nel 1861 usciva in Parigi presso l'editore Bourdillat un volume di oltre 350 pagine dal titolo L'expédition des deux Siciles. Souvenirs personnels in cui trovavano posto, sia pure rimaneggiati, gli articoli pubblicati dalla «Revue des deux mondes» tra marzo e maggio di quello stesso anno nonché alcuni resoconti giornalistici usciti anonimi l'anno prima su «L'Opinion nationale» e veniva utilizzata parte di una documentazione di prima mano ( proclami, circolari, fotografie ) oggi conservata nei Mss 3723 della Bibliothèque de l'Institut de France, a Parigi. Ne era autore Maxime Du Camp.

L'opera che conobbe una seconda edizione nel 1881, figura raramente tra le testimonianze di maggior rilievo sulla spedizione dei Mille, forse a causa del gran numero di altri scritti che, in quello stesso anno, furono pubblicati da autori ben noti quali Marc Monnier e Louise Colet. Del primo si ricorda Garibaldi. Histoire de la conquête des deux Siciles (Paris, M. Lévy) e della Colet Naples sous Garibaldi (Paris, Dentu). Ma soprattutto perché, fin dall'anno precedente, era uscita sui giornali francesi una serie di corrispondenze, poi raccolte in volume con il titolo Les Garibaldiens, di uno scrittore la cui opera da tempo era legata al nome di Garibaldi, Alexandre Dumas². Malgrado i limiti ancor oggi riconosciuti dalla critica (penso non solo al giudizio di Benedetto Croce, ma anche a quello, assai più recente, del Pécoud), che rimprovera

(1) Parte di questo articolo è stata oggetto di una conferenza tenuta all'Accademia Ligure di Scienze e Lettere il 21 ottobre 2011 nell'ambito delle celebrazioni per i 150 anni dell'unità d'Italia.
(2) Sui rapporti tra Dumas e Garibaldi cfr. il nostro De l'bistoire au mythe.Garibaldi vu par A.Dumas, in Elaborazioni poetiche e studi di genere, Alessandria, Edizioni dell'Orso, 2010, pp. 795-806. 
allo scrittore francese il suo eccessivo protagonismo e la tendenza a costruire un'immagine mitica dell'eroe dei due mondi ${ }^{3}$, la testimonianza di Dumas ha costituito un punto di riferimento indiscusso per la storia del nostro Risorgimento.

In Italia, soltanto nel 1963 L'expédition è stata tradotta da L. De Rosa e pubblicata dall'editore Cappelli di Bologna, con un'ampia e documentata introduzione di G. Macera. Nel 1976 Maria Gabriella Adamo ha dato un'edizione del testo francese nel quale, tuttavia, erano stati operati alcuni tagli, laddove la curatrice riteneva trattarsi di episodi «narrati di riporto o d'interesse anneddotico» ${ }^{4}$. Grande attenzione meritano tuttora l'ampia e documentata introduzione e le numerose note al testo.

La decisione di Du Camp di raggiungere Garibaldi in Sicilia nell'agosto del 1860 nasce sicuramente dalle notizie che giungono dall'Italia e in particolare dall'entusiasmo che suscitano in Francia gli articoli di Dumas con la descrizione della battaglia di Milazzo pubblicati da «La presse», «Le siècle», «Le Constitutionnel», «Le sémaphore de Marseille» ${ }^{5}$, ma anche dalla particolare situazione dello scrittore che sta attraversando una dolorosa crisi sentimentale e, per una serie di equivoci, ha visto mettere in discussione la sua amicizia con Flaubert ${ }^{6}$. Dopo aver invano cercato di convincere Gautier ad accompagnarlo ${ }^{7}$, Du Camp parte il 13 agosto 1860 da Genova, dove si è unito a un gruppo di patrioti ungheresi che gli saranno compagni durante tutta la spedizione, tra cui Téléki e Türr che lo nominerà suo colonnello. Egli arriva dunque in Sicilia proprio nel momento in cui, conquistata la maggior parte dell'isola, i Garibaldini si apprestano a sbarcare in Calabria. La sua partecipazione all'impresa dei Mille si svolge prevalentemente sul continente, secondo un itinerario che lo condurrà da Pizzo, Scilla, Cannitello a Bagnara Calabra, quindi Palmi, Mileto, Maida, Soveria, Marcellinara, Catanzaro, Tiriolo, Rogliano, Cosenza, Castrovillari,Castelluccio, Lauria, Lagonegro, Eboli, Salerno, fino a Napoli che raggiunge il 9 settembre, due giorni dopo l'ingresso trionfale di Garibaldi in città, e poi Caserta, il Volturno e Capua. Malgrado il titolo e seppur divisa in tre parti: La Sicile, Les Calabres, Naples et les avant-postes de Capoue, la seconda parte dell'Expédition che consta di ben otto capitoli (circa 150 pagine nell'edizione del 1861) ne costituisce il nucleo principale e per certi versi, come vedremo, il più originale.

L'Expédition si apre con la descrizione di Genova alla vigilia dell'imbarco di Du Camp per la Sicilia:

Les volontaires, reconnaissables à leur chemise rouge, marchaient bruyamment dans les étroites rues de Gênes au roulement des tambours... Dans le port, des bâteaux à vapeur chauffaient, qu'on chargeait de troupes et qui partaient pour leur destination pendant que les volontaires poussaient ce cri de ralliement qui devait conquérir un royaume: Vive l'Italie, toute et une $!^{8}$

\section{E Du Camp osserva:}

Ce mouvement n'en était pas moins très imposant par son unanimité; chaque province tenait en honneur d'envoyer des soldats rejoindre l'expédition libératrice; les vieilles haines

(3) Cfr. B.Croce, Alessandro Dumas a Napoli nei primi anni della nuova Italia, Bari, Laterza,1927, pp. 346-352 e G. PÉCOUD, Introduzione a A. DUMAS, Viva Garibaldi, Torino, Einaudi, 2004, pp. VII-XxxI.

(4) M. Du Camp, L'Expédition des Deux-Siciles, Saggio introduttivo di M.G. AdAmo, Reggio Calabria, Edizioni Parallelo 38,1976, p. 160.

(5) Sono le Lettres à Carini, già destinate a giornali italiani e pubblicate sui giornali francesi nel luglio-agosto 1860. Cfr. A. Dumas, Viva Garibaldi. Une Odyssée en 1860, présenté et annoté par
Cl. Schopp, Paris, Fayard, 2002, p. 448.

(6) Cfr. G. De Senneville, Maxime Du Camp. Un spectateur engagé du XIX siècle, Paris, Stock, 1996, pp. 249-259.

(7) Cfr. M. Du Camp, Souvenirs littéraires, texte conforme à l'édition de 1892, Paris, Aubin, 1994, pp. 464-465.

(8) M. Du Camp, Expédition des Deux-Siciles, Paris, Librairie nouvelle, A. Bourdillat et C. Editeurs, 1861, p. 8. Tutte le successive citazioni fanno riferimento a questa edizione 
provinciales, les amours-propres municipaux, qui jadis avaient fait tant de mal à la nation italienne, s'oubliaient en une seule pensée; ces anciens petits Etats, qui s'étaient autrefois épuisés à se guerroyer les uns contre les autres, réunissaient aujourd'hui leurs efforts pour arriver quand même à la formation d'une patrie commune. (p. 9)

C'è non poca retorica quando il Du Camp conclude: «Ces efforts n'auront pas été vains: tout verbe devient chair, et l'Italie sera, parce qu'elle a voulu l'être»; non dimentichiamo tuttavia che le sue parole ripropongono l'immagine che la Francia e l'Europa ebbero in quegli anni del nostro Risorgimento.

In questo quadro s'inserisce la figura di Garibaldi. Il primo incontro di Du Camp con il Generale avviene in un battello che lo porta da Palermo a Messina e sul quale sale anche Garibaldi. Questi stringe la mano a tutti, dice a ciascuno una parola gentile, poi si chiude nella sua cabina. Un po' più tardi Du Camp sente qualcuno cantare: «Ecouté par les matelots, au milieu des officiers, en face du capitaine anglais qui le regardait bouche béante Garibaldi chantait. Ce n'était alors ni le dictateur, ni le général en chef d'une armée révolutionnaire; c'était un bon compagnon qui profitait de ses loisirs pour se réjouir avec ses amis». Fu così, conclude Du Camp, che sentii Garibaldi cantare la bella romanza napoletana: «Ti voglio bene assai, ma tu non pensi a me» (p. 24).

Sarà soltanto durante la battaglia del Volturno che Du Camp vedrà nuovamente Garibaldi e in quell'occasione è il ritratto, oltre che di un uomo semplice, di un coraggioso combattente che ci viene proposto:

Il avait au hasard pris le premier cheval rencontré... coiffé de son petit chapeau noir hongrois, vêtu de la chemise rouge et du pantalon gris si connus, il excitait à son passage de tels cris d'enthousiasme qu'ils faisaient trembler la terre et couvraient le bruit du canon. (p. 316)

L'ultimo incontro di Du Camp con Garibaldi avviene alla vigilia della partenza per Caprera. Garibaldi si affaccia al balcone della Foresteria ove si erano riuniti a pranzo con il generale Türr, gli ufficiali della legione ungherese e parla alla folla che lo acclama. Il suo discorso che Du Camp riporta per intero (l'unica analoga testimonianza la ritroviamo soltanto in Monnier'), contiene un attacco diretto al potere temporale della Chiesa e la rivendicazione della propria fede cristiana: «Le pape-roi méconnaît le Christ, il méconnaît sa religion. Personne en entendant mes paroles, personne ne confondra le papisme et le christianisme, la religion de la liberté avec la politique de la servitude». E concludendo il suo discorso con un invito a dare una sana e forte educazione al popolo, dalla quale dipende la sua libertà, «Garibaldi se retourna vers moi en disant: Cela était nécéssaire» (p. 347).

In altre pagine dell' Expédition Du Camp aveva già mostrato di non ignorare il problema che rappresentava per la realizzazione dell'unità d'Italia lo Stato Pontificio e la posizione che in questi anni aveva assunto il governo francese. Se, da una parte, si dice convinto, come scriverà nei Souvenirs, della «connivence du gouvernement français à l'unification de l'Italie», sicché, con la sua partecipazione alla spedizione dei Mille «je ne me trouvais en opposition avec l'action diplomatique de la France» ${ }^{10}$, dall'altra, egli non ignora l'intenzione di Garibaldi di «marcher d'emblée sur Rome, l'enlever par un coup de main, la déclarer capitale du royaume péninsulaire et y proclamer Victor-Emmanuel roi d'Italie» (p. 255). Ma, nel 1860, i tempi non sono ancora maturi: la Francia è a Roma, non tanto per motivi religiosi, ma per esigenze politiche, 
e qualunque tentativo di conquistare la Capitale da parte di Garibaldi sarebbe destinato all'insuccesso, «le jour viendra, conclude profeticamente Du Camp, et peut-être n'est-il pas éloigné, où la France pourra laver ses mains innocentes en présence de toutes les nations et détourner sa face, afin que les destinées s'accomplissent. Ce jour là, s'élevant encore au-dessus de ses gloires passées, Rome deviendra la capitale de la liberté, comme elle a été celle de la force brutale et celle de l'absolutisme» (p. 257).

La consapevolezza di quanto sia complicata e delicata, specie dal punto di vista francese, la questione romana, spiega forse, anche se non giustifica, quanto Du Camp scrive a Flaubert in una lettera inviata da Napoli il 18 ottobre 1860. All'amico che si stupisce del suo lungo silenzio Du Camp, dopo aver decantato la bellezza del paesaggio calabro, scrive, con il solito tono scanzonato e spesso volgare che caratterizza la loro corrispondenza: «J'ai cru un instant que cet imbécile de Garibaldi, allait tout de même essayer d'attaquer Rome; quelle tripotée il aurait reçue, et comme ça aurait avancé les affaires de la liberté du monde. Heureusement qu'il a renoncé à ce beau projet, ce qui m'étonne de son cerveau qui n'est pas fort $\gg^{11}$.

Se il giudizio su Garibaldi politico è ingeneroso nella sua severità, altro è da parte di Du Camp il riconoscimento dei meriti del condottiero e del suo prestigio presso la gente. Per tutto il tragitto lungo la Penisola e fino a Napoli,Garibaldi precede il gruppo di combattenti di cui Du Camp fa parte, ma egli è presente nel cuore di tutti coloro che lo seguono nella sua impresa. La sua popolarità nasce dalla sua stessa natura dal momento che «Garibaldi est un simple au bon sens du mot. Porté par un immense amour de sa patrie, il a accompli naïvement des œuvres énormes, ne tenant jamais compte des obstacles, ne voyant que le but auquel il marche droit, sans que la possibilité de fléchir lui soit même venue à l'esprit. Son instruction paraît médiocre, son intelligence ordinaire, son esprit assez crédule; mai il a un grand cœur. Il croit à l'Italie, il croit à sa mission» (p. 29) Ecco perché «il a été admirablement secondé par la nation italienne. Tout ce grand peuple, issu de la même race, parlant la même langue, professant la même religion, n'ayant entre les différentes familles qui le composent que des frontières diplomatiques, est fatigué outre mesure des divisions arbitraires... il est justement las d'être considéré comme un troupeau... il veut rassembler ses membres dispersés, il veut se réunir lui-même, il veut être un» (p. 30).

Garibaldi si è fatto interprete di questa esigenza: «Il a révolutionné les $\mathrm{Ca}$ labres par les Calabrais et Naples par les Napolitains» (p. 230); di qui il giudizio sostanzialmente positivo anche se non volto alla mitizzazione del personaggio, di Du Camp: «Seul peut-être parmi ceux que j'ai rencontré, Garibaldi ne m'a fait éprouver aucune déception» (p. 28).

Aiuta a meglio comprendere l'atteggiamento di Du Camp nei confronti di Garibaldi quanto leggiamo, sempre nell' Expédition, a proposito di Mazzini ${ }^{12}$. Du Camp che definisce il patriota genovese «un homme célèbre dans l'histoire révolutionnaire de notre temps», scrive, allineandosi anche con questo giudizio alla politica portata avanti in quegli anni dalla Francia di Napoleone III: «Je n'aime pas ses doctrines et, quoique j'ai souvent admiré la fermeté de ses convictions résistant à l'exil, à la ruine, à l'emprisonnement, aux persécutions de toutes polices imaginables, je crois que sa route n'est pas la bonne route, et qu'au lieu d'être un profète il est tout au plus un précurseur».

(11) M. Du CAMP, Lettres inédites à G.Flaubert, Introduzione e note di G.BOnACCORSO e R.M. DI Stefano, Messina, EDAS,1978, pp. 226-230.

(12) Com'è noto Mazzini era stato espulso dalla
Francia nel 1835 a seguito della tentata rivolta in Savoia e nel 1849 era stato uno dei triumviri della Repubblica Romana. 
E tuttavia condanna le contestazione di cui Mazzini è stato oggetto a Napoli per il suo atteggiamento al momento del plebiscito in favore di Vittorio Emanuele e conclude:

Je crois qu'à Naples il fut admirable de dévouement et d'abnégation; avant d'aimer une forme politique, il aime sa patrie, il est Italien plus encore que républicain (p. 248) .

Lungo tutto il percorso attraverso la Calabria, la Basilicata, la Campania Du Camp incontra una popolazione che ha accolto con entusiasmo Garibaldi e i Garibaldini:

Partout on accourait: du haut des montagnes les paysans armés venaient au-devant de lui et l'entouraient; les villes se pavoisaient à son approche, et les habitants restaient debout éveillés pendant des nuits entières, suspendus par l'attente de cet homme qui passait plus rapide et plus fort que le tonnerre. (p. 210)

Poco dopo lo sbarco sul continente è la volta di un gruppo di uomini di Scilla:

Ils sont vigoureux, bruns de face et larges des épaules, chaussés d'espadrilles, coiffés d'un haut bonnet de laine bleue à la marinière, les cuisses serrées dans une culotte presque collante retenue par une large ceinture où brille le manche d'un couteau, ils vont d'un pas régulier et ferme, portant sur l'épaule un long fusil qui, sauf la crosse, pareille aux vieilles crosses françaises, ressemble aux vieux fusils albanais. Ils nous saluent d'un beau regard clair quand nous passons près d'eux, et crient en patois: Viva la Talia una! - Où vas-tu? demandai-je à l'un d'eux. - A Venise! (p. 112)

E come dimenticare l'accoglienza di Napoli: «Un flot diapré et hurlant montait et descendait la rue de Tolède; tous ceux qui avaient pu trouver une loque rouge, casaque, châle ou rideau, s'en étaient affublés, et, levant les bras, vociférant, agitant des bannières, s'embrassant, riant, pleurant s'en allaient acclamer le dictateur» (p. 243).

Attraversando le regioni meridionali della nostra Penisola, facendo tappa in diversi paesi, Du Camp dedica molte pagine alla descrizione del paesaggio: la fertile pianura nei dintorni di Bagnara, le rupi dell'Aspromonte, i greti sassosi dei torrenti, le vie che s'inerpicano sui fianchi scoscesi delle montagne, i piccoli villaggi abbarbicati sui picchi più alti. È lo stesso viaggiatore che della sua esperienza in Normandia, in Egitto, in Medio Oriente ha lasciato ampia testimonianza nelle sue opere, il fotografo di Le Nil che guarda, con occhio attento, i luoghi che attraversa, affascinato dalla bellezza e dalla varietà di una regione lontana dai consueti itinerari del tradizionale voyage en Italie.

$\mathrm{Ma}$, soprattutto, Du Camp rievoca gli avvenimenti che si sono svolti in quei luoghi negli ultimi cinquant'anni della loro storia. Così, passando per Pizzo, una città che giudica di tendenze non proprio liberali, ricorda la cattura e la morte di Gioacchino Murat (p. 199) e a Cosenza non può non evocare l'infausta vicenda dei fratelli Bandiera le cui spoglie, proprio al passaggio delle truppe garibaldine, ricevono giusta sepoltura. Du Camp in questa occasione racconta di aver incontrato a Smirne il barone Bandiera, ammiraglio della marina austriaca i cui figli «venaient de se jeter dans une généreuse aventure», cita i rapporti dei due giovani con Mazzini e la Giovane Italia, rievoca con dovizia di particolari il loro tentativo rivoluzionario, la sconfitta, il processo e la morte (pp. 199-203). A Sapri non può non parlare dell'infausta avventura di Carlo Pisacane che «blessé, désarmé, après s'être loyalement rendu, fut assommé à coups de bâton et déchiré à coups de fourche comme un loup enragé» (p. 153). Quella stessa popolazione che accoglieva Garibaldi come un santo (a Palermo la sua 
immagine figurava spesso accanto a quella della Madonna) era capace di grandi violenze e atrocità, talvolta soltanto per viltà, in altri casi in vista di qualche possibile vantaggio. Basti pensare al recente ammutinamento delle truppe borboniche e alla morte del Generale Briganti, quando, dopo averlo ucciso, «la troupe enivrée du meurtre se jeta à coup de baïonnette sur son ancien général et le mit en pièces». Poi, dopo averlo spogliato, «ces sauvages... tirant par ses pieds nus le pauvre cadavre, ils l'accablèrent d'outrages sans noms, lui arrachant les cheveux et les moustaches, enfonçant dans les orbites des capsules auxquelles ils mettaient le feu, lui traversant le nez avec des épinglettes» (p. 144).

Come spiegare tanta crudeltà e lo stesso fanatismo religioso che caratterizza le popolazioni delle regioni meridionali che attraversa se non con l'ignoranza in cui sono state tenute dal governo borbonico con la complicità della Chiesa?

A Cannitello, accolto nella casa del sindaco, Du Camp domanda se in paese c'è una scuola, al che il curato che è anche il fratello del sindaco risponde: «Ah! Dieu merci, non!» e Du Camp conclude con tristezza: «Dans presque tous les villages du royaume de Naples j'ai fait la même question et obtenu la même réponse» (pp. 105106).

Anche a Castelluccio, un paese di oltre cinquemila abitanti, c'è una sola scuola, frequentata da non più di otto o dieci bambini. Un abitante del luogo che ha viaggiato e conosce il mondo spiega le ragioni di tanta diffusa ignoranza: «A ces pauvres gens l'instruction cause une sorte de terreur superstitieuse que les prêtres entretiennent avec soin, car l'ignorance de tous leur rend très facile la tâche de les diriger. Un homme qui sait lire et qui lit est mal vu, soupçonné d'appartenir à des sociétés secrètes, traité d'esprit fort, accusé d'impiété». Per non parlare degli avvocati che secondo l'opinione di un notabile del posto dovrebbero esser tutti eliminati perché «tous les avocats sont mazziniens forcément et sans exceptions». Sicché in certi comuni «les curés refusent l'absolution aux mères qui envoient leurs enfants aux collèges de Naples» (p. 216).

Forse Du Camp esagera un po', forse è l'ex-allievo di un celebre liceo parigino che si esprime in queste pagine riprendendo anche una serie di luoghi comuni - la descrizione della sporcizia delle città, il fanatismo religioso che diventa idolatria - che troviamo in molti racconti di viaggiatori stranieri nel meridione d'Italia in quegli anni. Ma occorre ricordare a questo proposito che, tornato a Napoli nel 1862, Du Camp osserva, con un certo ottimismo, che la città ha già subito una trasformazione radicale, proprio grazie alla creazione di molte scuole e all'obbligo dell'istruzione imposto dal governo italiano ${ }^{13}$.

Il 9 novembre, dopo la battaglia del Volturno, l'assedio di Capua e l'incontro con Vittorio Emanuele, Garibaldi lascia Napoli per Caprera. Due giorni dopo anche Du Camp s'imbarca sul 'Céphise' per far ritorno in Francia.

L'ultimo episodio di cui è stato spettatore e di cui ci parla nell'Expédition è la mancata rivista delle truppe garibaldine da parte di Vittorio Emanuele.

«Le 5 novembre, scrive Du Camp, tous les garibaldiens qui étaient casernés à Naples reçurent l'ordre de se rendre à Caserte, où le roi devait les passer en revue; pendant toute la journée du 6 ils l'attendirent vainement; il ne daigna point passer devant nos 'bandes' ...». E, dopo aver ricordato che il giorno seguente Vittorio Emanuele celebrava con fanfare e medaglie i soldati piemontesi mentre solo alcuni ufficiali in camicia rossa assistevano dall'alto di un balcone alla parata militare, conclude

(13) Cfr. M.G. AdAmo, Maxime Du Camp a Napoli nel 1860 e nel 1862, Comunicazione presentata su Napoli e la Francia, Naples et la France, Napoli, 1979, pp. 18-19. 
con amara ironia: «Il était naturel qu'ayant conquis la Sicile, délivré les Calabres, dispersé les troupes bourboniennes, pris Naples, défendu à outrances leurs positions devant Capoue et gagné seuls la bataille du Vulturne, il était naturel qu'ils fussent éloignés le jour du triomphe» (p. 349).

Molti anni sono passati (siamo nel 1880) quando Du Camp torna a parlare della sua avventura garibaldina. Il nostro autore è ormai uno scrittore affermato, è stato nominato senatore da Napoleone III, è diventato Accademico di Francia, ma ha visto anche la fine del Secondo Impero, la disastrosa guerra franco-prussiana, le tragiche giornate della Commune. L'unità d'Italia, di quell'Italia che, scrive Du Camp, «j'ai toujours aimée et pour laquelle j'ai été assez heureux de combattre au temps de ma jeunesse ${ }^{14}$ si è ormai pienamente realizzata. Grande merito ha avuto soprattutto in questo l'intelligente opera di Cavour a cui Du Camp riconosce di aver saputo interpretare «l'âme de l'Italie», sicché «tout ce qu'il a fait il l'a fait avec le concours de sa nation... Il était le porte-voix, le porte glaive de son peuple, et c'est ce qui l'a fait invincible» ${ }^{15}$.

E la spedizione dei Mille? Essa resta ancora «la belle aventure». Scrive Du Camp nei suoi Souvenirs:

Vit-on jamais pareil soulèvement, si spontané, si universel? J'en doute. Les villages, les villes se précipitaient au-devant de nous et l'armée royale disparaissait à notre approche comme un vol d'oiseaux effarouchés... De Reggio à Naples nous avons marché en corps, en groupes, isolés, sans avoir à tirer un seul coup de fusil.

Quanto a Garibaldi «Garibaldi a aimé son pays avec passion, il en a partagé les illusions; ... le patriotisme est une vertu si belle, qu'elle doit faire excuser bien des fautes, bien des folies. Garibaldi eut un tort, un tort irréparable que l'histoire ne lui pardonnera jamais. Il n'est pas mort à temps». Il momento culminante della sua carriera lo ha vissuto quando ha consegnato a Vittorio Emanuele l'Italia: «depuis cette heure il n'a fait que décroître, il marche dans sa gloire éteinte... Garibaldi vivant est pour toujours tombé sur la cime d'Aspromonte, il ne s'en est plus relevé» ${ }^{16}$.

A distanza di anni, l'atteggiamento di Du Camp non è poi molto cambiato. Lontano ormai per lunghi periodi da Parigi, nel sereno, volontario esilio di Baden-Baden, senza dubbio meno partecipe delle attuali vicende politiche del suo Paese e dell'Europa, Du Camp ignora il generoso intervento di Garibaldi e il contributo dei garibaldini in difesa della Francia nel Settanta. Egli resta legato al ricordo della spedizione dei Mille ma non sa, o non vuole dimenticare, né l'Aspromonte né Mentana.

CECILIA RIZZA

(14) Cfr. M. Du Camp, Lettera ad A. De Gubernatis dell' 8 marzo 1875 in P. CIUREANU, Lettere di J. Barthélemy Saint-Hilaire e M. Du Camp ad A. De Gubernatis, «Studi francesi», n. 50, maggio-agosto
1973 , p. 273.

(15) M. Du Camp, Souvenirs littéraires, pp. 465466.

(16) Ibid., pp. 469-470. 